OPEN ACCESS

Edited by:

E. Christien Michael Parsons, University of Glasgow,

United Kingdom

Reviewed by:

Patricia Briones-Fourzan,

Universidad Nacional Autónoma de México, Mexico

Tammy Robinson-Smythe

Stellenbosch University, South Africa

*Correspondence:

Lynette H. L. Loke

lynetteloke@gmail.com;

dbsloke@nus.edu.sg

Specialty section:

This article was submitted to

Marine Conservation

and Sustainability,

a section of the journal

Frontiers in Marine Science

Received: 04 November 2018 Accepted: 14 January 2019

Published: 30 January 2019

Citation:

Loke LHL, Heery EC, Lai S, Bouma TJ and Todd PA (2019)

Area-Independent Effects

of Water-Retaining Features on Intertidal Biodiversity on

Eco-Engineered Seawalls

in the Tropics. Front. Mar. Sci. 6:16.

doi: 10.3389/fmars.2019.00016

\title{
Area-Independent Effects of Water-Retaining Features on Intertidal Biodiversity on Eco-Engineered Seawalls in the Tropics
}

\begin{abstract}
Lynette H. L. Loke ${ }^{1 *}$, Eliza C. Heery ${ }^{1}$, Samantha Lai ${ }^{1}$, Tjeerd J. Bouma ${ }^{2,3}$ and
\end{abstract}
Peter A. Todd ${ }^{1}$

${ }^{1}$ Experimental Marine Ecology Laboratory, Department of Biological Sciences, National University of Singapore, Singapore, Singapore, ${ }^{2}$ Department of Estuarine and Delta Systems, Royal Netherlands Institute for Sea Research (NIOZ), Utrecht University, Yerseke, Netherlands, ${ }^{3}$ Faculty of Geosciences, Department of Physical Geography, Utrecht University, Utrecht, Netherlands

Over the last decade there has been a global effort to eco-engineer urban artificial shorelines with the aim of increasing their biodiversity and extending their conservation value. One of the most common and viable eco-engineering approaches on seawalls is to use enhancement features that increase habitat structural complexity, including concrete tiles molded with complex designs and precast "flowerpots" that create artificial rock pools. Increases in species diversity in pits and pools due to microhabitat conditions (water retention, shade, protection from waves, and/or biotic refugia) are often reported, but these results can be confounded by differences in the surface area sampled. In this study, we fabricated three tile types $(n=10)$ : covered tile (grooved tile with a cover to retain water), uncovered tile (same grooved tile but without a cover) and granite control. We tested the effects of these tile types on species richness $(S)$, total individual abundance $(N)$, and community composition. All tiles were installed at $0.5 \mathrm{~m}$ above chart datum along seawalls surrounding two island sites (Pulau Hantu and Kusu Island) south of Singapore mainland. The colonizing assemblages were sampled after 8 months. Consistent with previous studies, mean $S$ was significantly greater on covered tiles compared to the uncovered and granite tiles. While it is implied in much of the eco-engineering literature that this pattern results from greater niche availability allotted by microhabitat conditions, we further investigated whether there was an underlying species-individual relationship to determine whether increases in $S$ could have simply resulted from covered tiles supporting greater $N$ (i.e., increasing the probability of detecting more species despite a constant area). The species-individual relationship was positive, suggesting that multiple mechanisms are at play, and that biodiversity enhancements may in some instances operate simply by increasing the 
abundance of individuals, even when microhabitat availability is unchanged. This finding underscores the importance of testing mechanisms in eco-engineering studies and highlights ongoing mechanistic uncertainties that should be addressed to inform the design of more biodiverse seawalls and urban marine environments.

Keywords: habitat complexity, ecological engineering, urban marine ecology, species-area relationship, speciesabundance relationship

\section{INTRODUCTION}

Growing coastal populations that are increasingly threatened by rising seas (Hinkel et al., 2014; Nicholls, 2015) are driving the construction of coastal defenses and flood protection, particularly in low-elevation coastal zones and vulnerable urban centers (Neumann et al., 2015). Ideally, coastal adaptation planning and defense systems should employ nature-based approaches, including "planned retreat" (pulling developments back from the present shoreline) and the creation or restoration of ecosystems that naturally protect against flooding, such as salt marshes and mangroves (Temmerman et al., 2013; Narayan et al., 2016). However, traditionally engineered artificial structures, such as seawalls, are pervasive, particularly in coastal cities, where sea level rise adaptation efforts primarily aim to protect the existing shoreline or advance the shoreline into adjacent marine habitats (Nicholls, 2011; Dafforn et al., 2015). These structures have considerable impacts on marine ecosystems (Bishop et al., 2017; Heery et al., 2017, 2018), yet are likely to remain a central component of coastal defense systems, either as the primary mode of flood protection or in combination with natural features (Bulleri and Chapman, 2009; Cheong et al., 2013). In response, there has been a global effort during the last decade to ecologically engineer hard artificial structures with the aim of improving their ecological value and alignment with conservation goals (Chapman and Underwood, 2011; Dyson and Yocom, 2015; Pioch et al., 2018). Ecological engineering solutions have already been applied to artificial shoreline projects in several cities, with various benefits to both marine ecosystems and human communities in urban areas (Arkema et al., 2017; Morris et al., 2018). Yet, for many of them, there have been few experimental studies that systematically explore precisely how they alter marine biota. A thorough understanding of the underpinning mechanisms through which ecological enhancements operate remains elusive.

Though ecological engineering of artificial structures can be used to meet a variety of objectives, its core environmental aims are often to enhance biodiversity and extend the conservation of native marine species to urban habitats that would otherwise be inhospitable (Loke et al., 2019). One of the most popular techniques for intertidal seawalls is to increase habitat structural complexity (Loke et al., 2014, 2017), for instance by removing pieces of the seawall to create recesses (Chapman and Blockley, 2009), drilling pits to form rock pools (Martins et al., 2010; Evans et al., 2016; Hall et al., 2018), and attaching "flower pots" and other concave enhancements (Browne and Chapman, 2011, 2014; Firth et al., 2016a; Waltham and Sheaves, 2018). These manipulations have repeatedly been shown to increase the richness and abundance of benthic organisms on intertidal seawalls (reviewed by Loke et al., 2019), and their effectiveness is frequently attributed to increased shade and moisture (i.e., increased niche availability) allotted by structurally complex microhabitat features (Firth et al., 2014, 2016b; Perkol-Finkel et al., 2018; Strain et al., 2018), which can reduce temperature fluctuations, minimize desiccation stress, and facilitate the recruitment of sessile fauna (Metaxas and Scheibling, 1993; Blockley and Chapman, 2006; Seabra et al., 2011; Firth et al., 2013). To date, the majority of related experiments in this area have been conducted in temperate areas (e.g., Browne and Chapman, 2011; Evans et al., 2016; Hall et al., 2018). The deleterious effects of temperature and desiccation are potentially a greater threat to tropical intertidal assemblages, and hence more data are needed from low latitude countries.

Discerning between the mechanisms that explain why structurally complex features enhance intertidal diversity is essential for ensuring that ecological engineering achieves its objectives and that unintentional consequences are avoided, particularly as these features are now being "scaled-up" to meet growing commercial demand for ecological enhancements. As has been widely suggested in past literature, creating additional microhabitat features may indeed increase biodiversity primarily by increasing niche availability. However, this has rarely been tested explicitly and various other explanations are often feasible. For instance, greater surface area in structurally complex treatments will lead to greater species richness (due to basic species-area relationships, SAR) and prove misleading if not carefully accounted for in eco-engineering studies. Alternatively, even when surface area is accounted for, an increase in niche availability is just one of the possible mechanisms through which enhanced biodiversity can arise. For example, microhabitat conditions associated with structurally complex features may instead support greater abundances/densities of individual organisms, which in turn could increase species richness simply by increasing the probability that more species will be detected as more individuals are sampled (henceforth referred to as "speciesindividual relationship"; see Fisher et al., 1943).

In this study, we explored multiple alternative explanations for biodiversity increases that have been well-documented in response to structurally complex eco-engineering features and informally linked to increases in niche availability. To determine whether, in fact, changes in microhabitat conditions (e.g., combined effect of increased water retention, shade, protection from waves and/or refugia) provide a parsimonious explanation of increased species richness on structurally complex features, we accounted for the potentially confounding effect of surface area on species richness in our statistical analyses. Furthermore, 


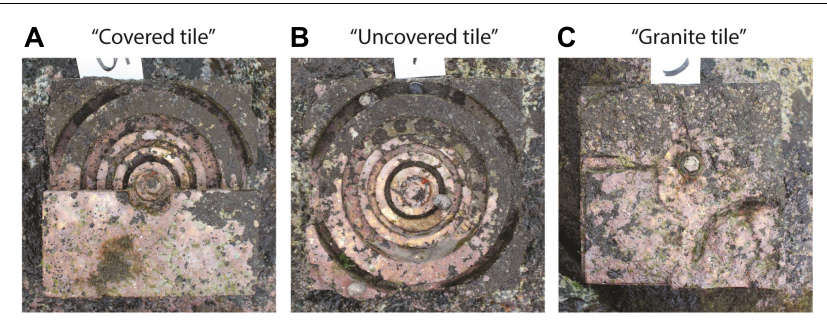

FIGURE 1 | Photographs (taken 6 months after deployment) of the three tile types tested in this study: (A) covered tile, (B) uncovered tile, and (C) granite control tile. Note the underlying tile design and surface area of $(\mathbf{A})$ and $(\mathbf{B})$ are the same.

to discern whether microhabitat conditions primarily altered biodiversity by increasing the availability of niches, or whether other non-niche-related mechanisms are also important, we conducted an a posteriori analyses of the relationship between the number of individuals $(N)$ and the number of species $(S)$.

We deployed tiles of the same underlying tile design (and thus level of topographic complexity) to seawalls in Singaporetreatments differed only in whether they had water retaining features (i.e., had a cover). Consistent with previous findings, we hypothesized that: (1) Covered tiles would host greater species richness $(S)$ compared to uncovered and granite (control) tiles; (2) Covered tiles would host a greater number of individuals (total abundance, $N$ ) compared to uncovered and granite tiles; and (3) Covered tiles would host assemblages that were compositionally different compared to uncovered and granite tiles. We additionally examined the species-individual relationship across treatments to assess whether there was evidence that microhabitat (niche) availability was likely the primary mechanism underlying these patterns. Note that by exploring the relationship between $S$ and $N$, we did not explicitly test any mechanism per se, rather, we used this analysis to rule out the possibility that increases in $S$ could be explained by increases in $N$, a pattern that would suggest that niche availability is not the only mechanism influencing biodiversity when surface area is held constant.

\section{MATERIALS AND METHODS}

\section{Experimental Design, Tile Fabrication and Deployment}

We fabricated three tile types: "covered" tile (grooved tile with a concrete cover plate to retain water, Figure 1A), "uncovered" tile (same grooved tile but without a cover, Figure 1B) and "granite" tile (Figure 1C). The concrete cover plate served to trap water and provide shade and protection from waves and predators in the "covered" tile treatment, but these components were not disentangled by our study. All tiles measured $200 \times 200 \times 60 \mathrm{~mm}$ (width $\times$ length $\times$ depth). The covered and uncovered tiles were made of concrete following the "complex-groove quarter tile" design used in Loke and Todd (2016) and were cast from silicone rubber molds using a 1:3 Portland cement to sand mix. Granite tiles, which served as a control, were made of broken granite slabs cemented onto a concrete base to mimic the surface of the surrounding granite seawall (for more details on how the tiles were constructed please refer to Loke and Todd, 2016). This was a procedural control and provided an idea of what the background seawall would support within the same timeframe given the same mounting system as the concrete tiles.

The tiles were deployed during low tides on 9-10 August 2010 along un-grouted, sloping $\left(\approx 30^{\circ}\right)$, granite rip-rap seawalls at two sites south of Singapore's mainland: Pulau Hantu $\left(1^{\circ} 13^{\prime} 34^{\prime \prime} \mathrm{N}\right.$, $\left.103^{\circ} 45^{\prime} 0^{\prime \prime} \mathrm{E}\right)$ and Kusu Island ( $\left.1^{\circ} 13^{\prime} 22^{\prime \prime} \mathrm{N}, 103^{\circ} 51^{\prime} 40^{\prime \prime} \mathrm{E}\right)$. Ten replicates of each tile type were attached onto the seawalls in random order using M8 stainless steel bolts (Figure 1). All tiles were installed flat along the sloping seawall (see Figure 1) $0.5 \mathrm{~m}$ above chart datum and spaced at least $2.0 \mathrm{~m}$ apart. Hence, there was a total of 60 tiles with "tile type" as a fixed factor and "site" as a random factor; i.e., 2 sites $\times 3$ tile types $\times 10$ replicates.

\section{Field Sampling and Laboratory Procedures}

The tiles were retrieved after 8 months. The colonizing benthic assemblage on the tiles was sampled; organisms on tile surfaces were scraped into trays immediately after retrieval. Benthic organisms colonizing the outside of the concrete cover plate on the covered tiles were not included, but those on the underside were. Even though the concrete plates provided additional substrate where they covered the grooves of the underlying tile, they also removed habitable area where they were in contact with the top of the ridges. The overall effect was that the covered tiles had slightly less surface area $\left(60 \mathrm{~cm}^{2}\right)$ available for colonization than the uncovered tiles. This amounts to a $6.9 \%$ difference that was accounted for in our statistical models (see section "Statistical Analyses"). Note that, although water retained by the covered tiles presumably provided greater threedimensional habitat for suspended organisms, these organisms were lost when covers were removed and thus were not among the organisms sampled. All specimens were stored in 70\% ethanol until they were manually sorted, counted and identified to species or morphospecies level (within known genera) except for polychaetes and amphipods, which were identified to family level, and algae, which were identified to functional group level following Loke et al. (2016).

TABLE 1 | Estimated parameters, standard errors, $z$-values, and $p$-values for the negative binomial mixed effects models; significant $p$-values are in bold.

\begin{tabular}{lcccc}
\hline Models & Estimate & SE & $\boldsymbol{z}$-value & $\boldsymbol{p}$-value \\
\hline Species richness $(S)$ & & & & \\
Intercept & 3.52 & 0.09 & 38.49 & $<\mathbf{0 . 0 0 1}$ \\
Uncovered tile & -2.09 & 0.10 & -20.35 & $<\mathbf{0 . 0 0 1}$ \\
Covered tile & -0.50 & 0.10 & -5.25 & $<\mathbf{0 . 0 0 1}$
\end{tabular}

Number of individuals $(\mathrm{N})$

Intercept

Uncovered tile

5.68

$-2.02$

0.85

0.17

6.72

$-11.87$

$<0.001$

Covered tile

$-0.49$

0.17

$-2.85$ 
A

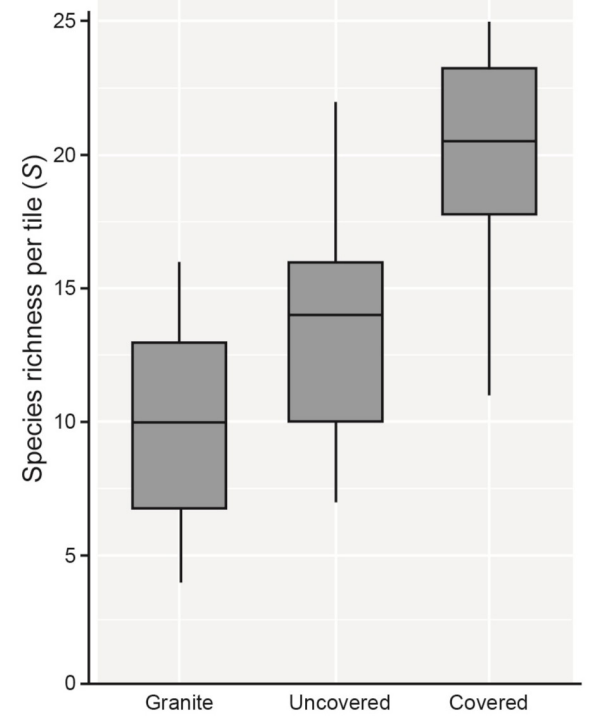

B

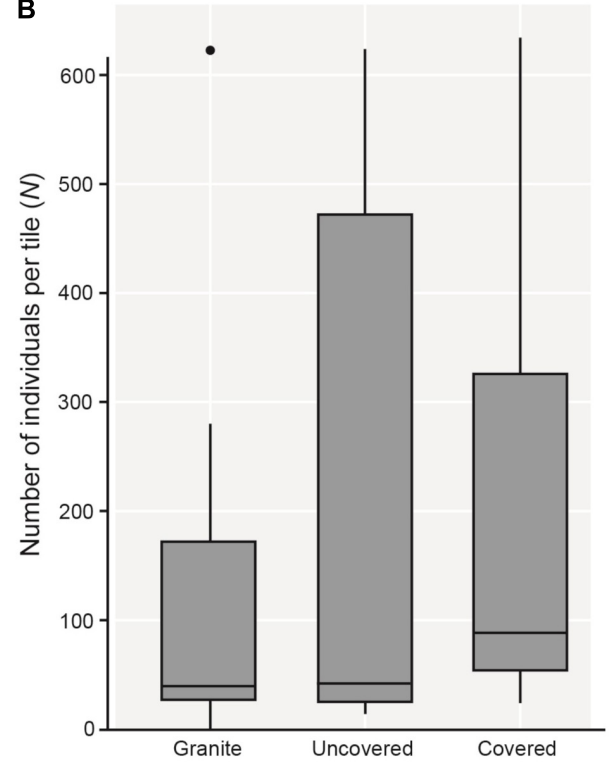

FIGURE 2 | Boxplots showing 5 and 95\% confidence intervals (whiskers), 25 and 75\% confidence intervals (boxes), and median of (A) species richness (S), and (B) total number of individuals (total abundance; $N$ ) found on each tile type.

\section{Statistical Analyses}

As some tiles were lost due to wave action, there were six covered tiles, seven uncovered tiles and six granite tiles remaining at Kusu Island, resulting in an unbalanced ANOVA design (no tiles were lost at Pulau Hantu). To avoid removing a large number of replicates to run a balanced analysis, we modeled species richness $(S)$ and number of individuals $(N)$ as counts using mixed effects models with negative binomial error terms to test for differences in $S$ and $N$ among tile types (Hypotheses 1 and 2, respectively). We selected the negative binomial error structure due to overdispersion in the Poisson models and included surface area as an offset in the model (Zuur et al., 2009). All models were constructed in R version 3.3.0 (R Development Core Team, 2016) with tile type as a fixed effect and site as a random effect. We then explored the potential species-individual relationship (i.e., relation between $S$ and $N$ ) via simple linear regression $[\operatorname{lm}()$ function in R]. Following the protocols outlined in Zuur et al. (2010), prior to our analyses we examined the data for overdispersion, non-linearity, heteroscedasticity, and presence of outliers.

To test for differences in community composition between tile types (Hypothesis 3), we used permutational distance-based multivariate analysis of variance (PERMANOVA; Anderson, 2001) to analyze the full resemblance matrix calculated on BrayCurtis similarities from log-transformed abundances with site as a random factor and tile type as a fixed factor. Due to highly significant differences between the communities among the sites, we then ran separate PERMANOVAs for each site. $P$-values were generated using 9999 unrestricted random permutations of residuals. Canonical analysis of principal coordinates (CAP) was applied to examine whether the communities on the three tile types could be separated and to explore the potential relationships between the individual species found and the canonical axes. The significance of the canonical relationship in each combination was tested using 9999 unrestricted random permutations of the transformed community data (Anderson et al., 2008). All multivariate analyses were performed using the PERMANOVA+ add-on for PRIMER v6 (Anderson et al., 2008).

\section{RESULTS}

A total of 8925 individuals representing 52 different faunal species and six algal functional groups were recorded. None of these species are unique to seawalls in Singapore (i.e., they are also found on natural rocky shores in Singapore; Lai et al., 2018) and none are known to be non-native (Jaafar et al., 2012; Tan et al., 2018).

As hypothesized, covered tiles hosted significantly greater $S\left(\chi^{2}=504.61, \mathrm{df}=2, p<0.001\right)$ and $N\left(\chi^{2}=153.65, \mathrm{df}=2\right.$, $p<0.001$ ) compared to uncovered and granite tiles (Table 1 and Figure 2). We also found that the variability in $N$ was due primarily to the dominance by a single species: Siphonaria guamensis at Kusu island (Figure 3). S. guamensis (the false limpet) was generally absent on tiles at Pulau Hantu (only a single individual recorded) but present in disproportionately large numbers at Kusu Island (319.1 \pm 154.8 individuals per tile; mean \pm SD) (Figure 3).

A plot of the abundance of $S$. guamensis alone revealed a similar trend to the plot of total individual abundance summarizing the whole dataset (i.e., Figure 3A vs. Figure 2B). On removing $S$. guamensis, the $N$ pattern resembled the $S$ pattern, with the greatest number of individuals found on covered 
A

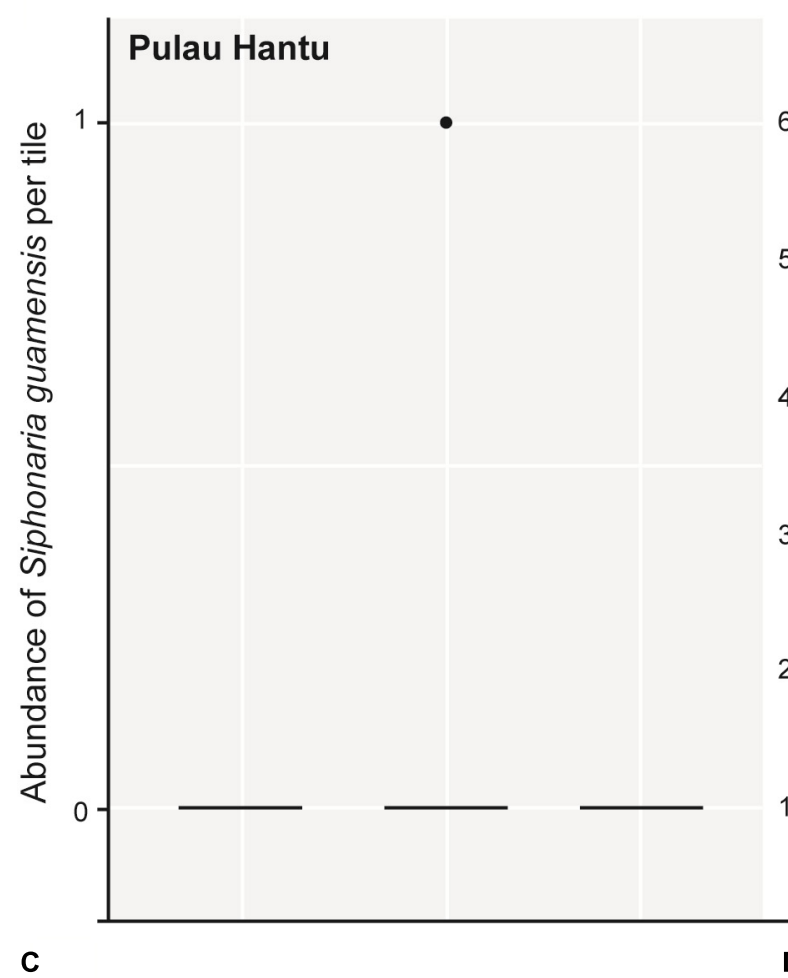

C

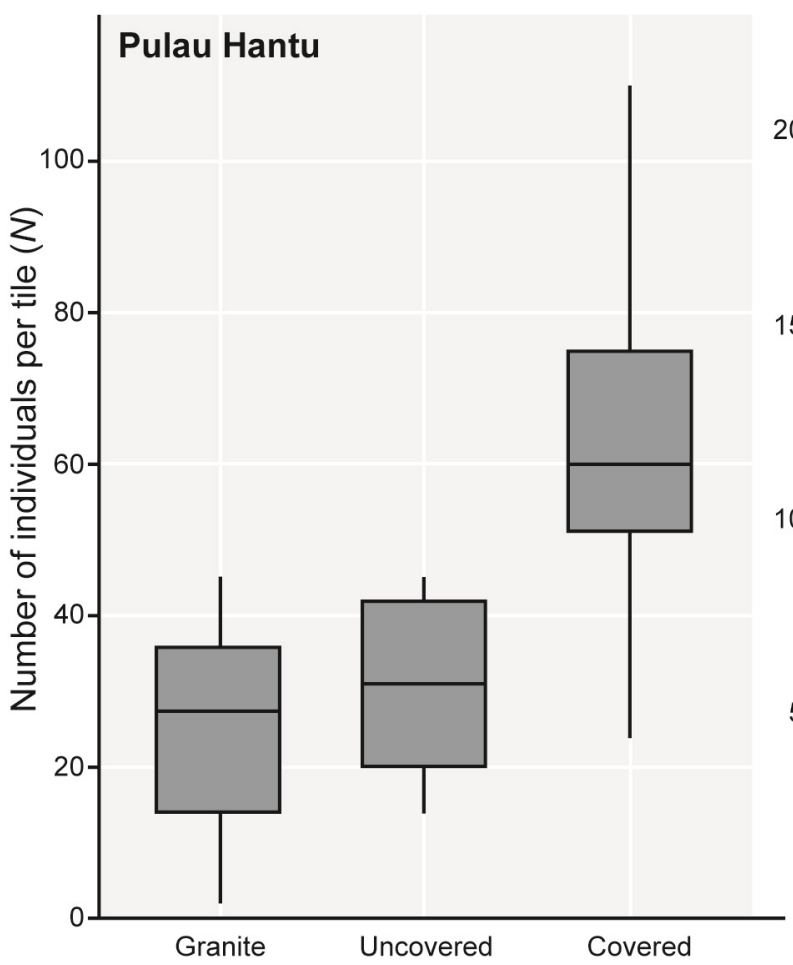

B

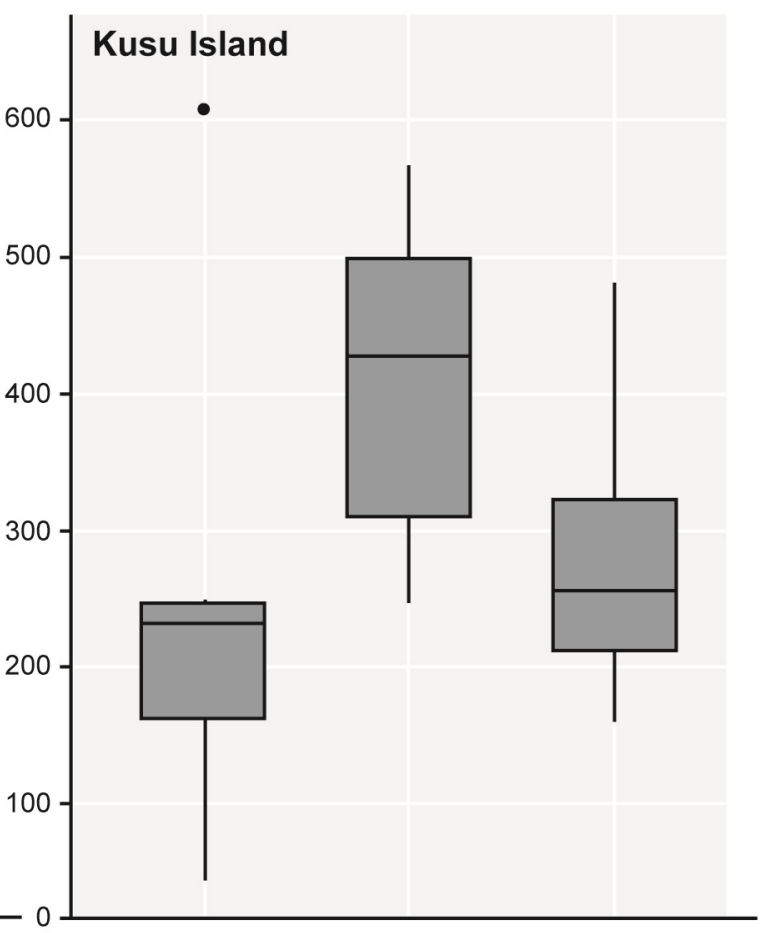

D

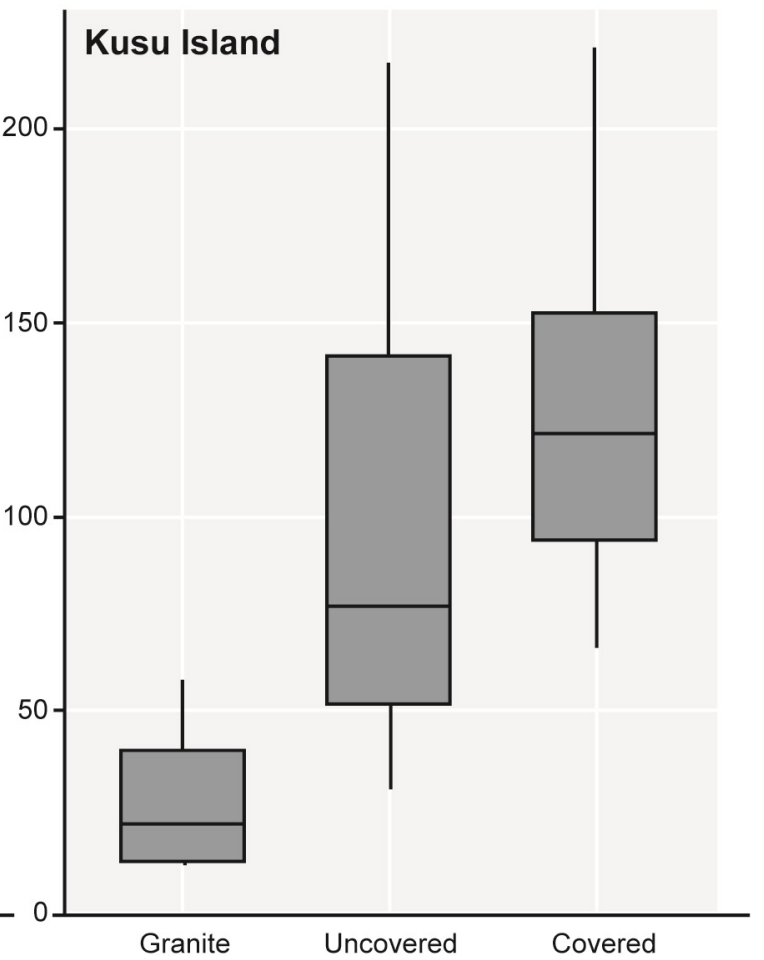

FIGURE 3 | Boxplots showing 5 and 95\% confidence intervals (whiskers), 25 and $75 \%$ confidence intervals (boxes), and median of the total abundance of (A) Siphonaria guamensis in each tile treatment at Pulau Hantu and (B) Kusu Island; (C) the total number of individuals ( $N$ ) of all 51 species in each tile treatment at Pulau Hantu and (D) Kusu Island after removing S. guamensis. 
tiles, followed by uncovered and granite tiles (Figures $3 \mathrm{~B}-\mathrm{C}$ vs. Figure 2A).

The simple linear regression between $S$ and $N$ revealed an overall significant positive relationship although the trend at each site differed, and was apparently non-linear at Kusu Island, again due to the disproportionately large abundances of S. guamensis (Figures 4A-B). As with the mixed effects model for total abundance, the removal of $S$. guamensis revealed highly significant positive linear relationships between $S$ and $N$ at both sites (Figures 4C-D).

Due to significant community differences between the assemblages at Pulau Hantu and Kusu Island (PERMANOVA; $\mathrm{df}=1,43, \mathrm{MS}=34172$, Pseudo- $F=26.3, P($ perm $)<0.001)$ we performed separate PERMANOVAs for each site. Results revealed that community composition differed significantly among the tile types at each site except between "uncovered" and "granite" tiles at Pulau Hantu (Table 2). The CAP analysis also showed a significant separation between the tile types at each site $(p<0.05$; Figure 5). Species commonly found under rocks, such as Barbatia amygdalumtostum and Isognomon legumen, characterized covered tiles.

\section{DISCUSSION}

Many of the ecological engineering efforts to improve biodiversity on urban structures have employed the use of water-retaining features (Firth et al., 2016a), but these features often differ in surface area from less structurally complex controls, and could be influencing species numbers. Without controlling for area, it is difficult to attribute increases in $S$ solely

TABLE 2 | PERMANOVA results based on Bray-Curtis dissimilarities of the relative abundances (pre-treated with $\log [X+1]$ transformation) of 56 different species in response to the tile type treatments.

\begin{tabular}{|c|c|c|c|c|c|c|}
\hline Source & df & MS & \multicolumn{2}{|c|}{ Pseudo-F } & $p$ (perm) & Unique perms \\
\hline \multicolumn{7}{|c|}{ Pulau Hantu } \\
\hline Tile type & 2 & 3666 & \multicolumn{2}{|c|}{2.1274} & 0.0084 & \multirow[t]{2}{*}{9913} \\
\hline Residual & 27 & 1723.2 & & & & \\
\hline Total & 29 & & & & & \\
\hline \multicolumn{7}{|c|}{ Kusu Island } \\
\hline Tile type & 2 & 2176.6 & \multicolumn{2}{|c|}{3.7061} & 0.0001 & \multirow[t]{3}{*}{9923} \\
\hline Residual & 16 & 587.3 & & & & \\
\hline Total & 18 & & & & & \\
\hline \multicolumn{2}{|l|}{ Groups } & \multicolumn{2}{|r|}{$t$} & $p$ (perm) & \multicolumn{2}{|c|}{ Unique perms } \\
\hline \multicolumn{7}{|c|}{ Pulau Hantu } \\
\hline \multicolumn{2}{|c|}{ Covered, uncovered } & \multicolumn{2}{|r|}{1.7646} & 0.0085 & \multicolumn{2}{|c|}{9418} \\
\hline \multicolumn{2}{|c|}{ Covered, granite } & & 1.779 & 0.0027 & \multicolumn{2}{|c|}{9402} \\
\hline \multicolumn{2}{|c|}{ Uncovered, granite } & \multicolumn{2}{|r|}{0.66005} & 0.9666 & \multicolumn{2}{|c|}{9384} \\
\hline \multicolumn{7}{|c|}{ Kusu Island } \\
\hline \multicolumn{2}{|c|}{ Covered, uncovered } & \multicolumn{2}{|r|}{1.4639} & 0.0023 & \multicolumn{2}{|c|}{460} \\
\hline \multicolumn{2}{|c|}{ Covered, granite } & & 2.2765 & 0.001 & 17 & \\
\hline Uncove & , granite & & 1.8583 & 0.0085 & 94 & \\
\hline
\end{tabular}

Significant $p$ (perm) values are highlighted in bold. to changes in microhabitat conditions-e.g., water retention, shade, protection from waves and/or biotic refugia-as speciesarea effects can dominate, as has been shown across nearly all systems and scales (Fahrig, 2013). Our study demonstrates experimentally that increased $S$ from water-retaining features is indeed a function of microhabitat provisioning (i.e., niche availability) to some extent, and this effect is independent of surface area. In fact, even though covered tiles had $6.9 \%$ less surface area than uncovered tiles, they supported greatest $S$. By ruling out surface area as the driver of enhanced biodiversity, this finding adds an essential piece to the mechanistic puzzle underpinning ecological enhancements of shorelines, and strengthens the foundation of ecological engineering as a discipline, ultimately aiding in its advancement.

We ruled out surface area as the driver of diversity patterns by comparing covered and uncovered tile treatments that offered the same structurally complex substrate for benthic organisms. As in previous studies, structurally complex tiles (both covered and uncovered tile treatments) were found to increase abundance and species richness compared to the less complex substrata that characterize seawalls (Loke and Todd, 2016). However, the added value from this study is exemplified by the differences observed between covered versus uncovered treatments. We observed higher $S$ on covered tiles compared with uncovered tiles. Covered tiles also supported greater $N$, and this effect was especially pronounced when the disproportionately abundant false limpet, S. guamensis, was removed (discussed further below). These findings are consistent with past studies (e.g., Chapman and Blockley, 2009; Browne and Chapman, 2011; Waltham and Sheaves, 2018), and suggest that incorporating additional microhabitats (e.g., shade and water retaining features) may be an effective strategy for biodiversity enhancement. More broadly, the findings lend weight to the idea that enhancing habitat (niche) complexity can and does play an important role in supporting more diverse communities on seawalls.

Water-retaining features are thought to enhance the diversity of intertidal biota through multiple mechanisms. Pits/pools are continually submerged, eliminating desiccation stress and other risks associated with emersion during low tide (Connell, 1972), and are rapidly colonized by a suite of taxa (Underwood and Jernakoff, 1984). They also provide greater shade than emergent surfaces, which dampens temperature variability (Seabra et al., 2011), reduces thermal stress (Williams, 1994; Bertness et al., 1999), and provides highly localized refugia for temperaturesensitive taxa, such as gastropods (Garrity, 1984) and newly recruited sessile invertebrates (Blockley and Chapman, 2006; Chapman and Blockley, 2009). This can lead to net increases in diversity (Blockley, 2007), even though reduced irradiance is limiting for some taxa, particularly primary producers (Goldberg and Foster, 2002). Mediated desiccation and temperature stress act in combination with a complex suite of additional abiotic and biotic variables (e.g., dissolved gas accumulation and decreasing pH during low tide, Huggett and Griffiths, 1986) to influence the composition of organisms in pits/pools, and community response to water-retaining features is therefore highly variable (Bugnot et al., 2018). The addition of a cover plate may also 


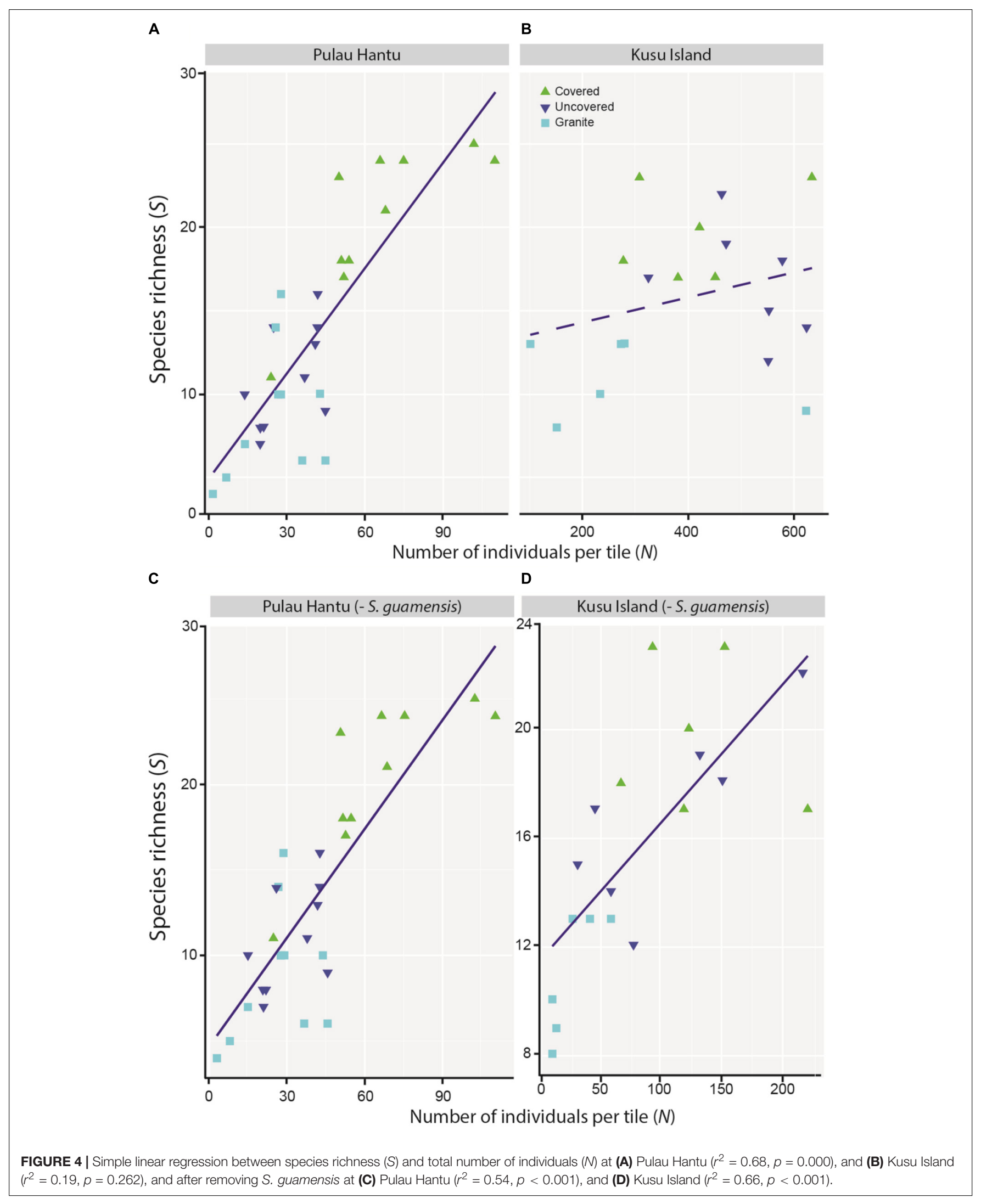


A

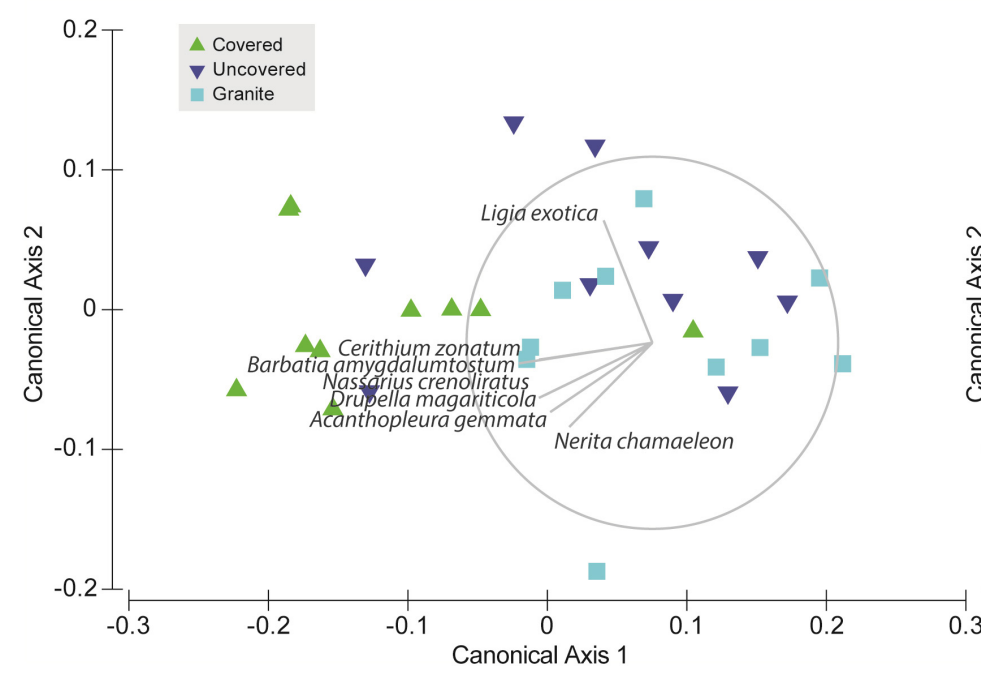

B

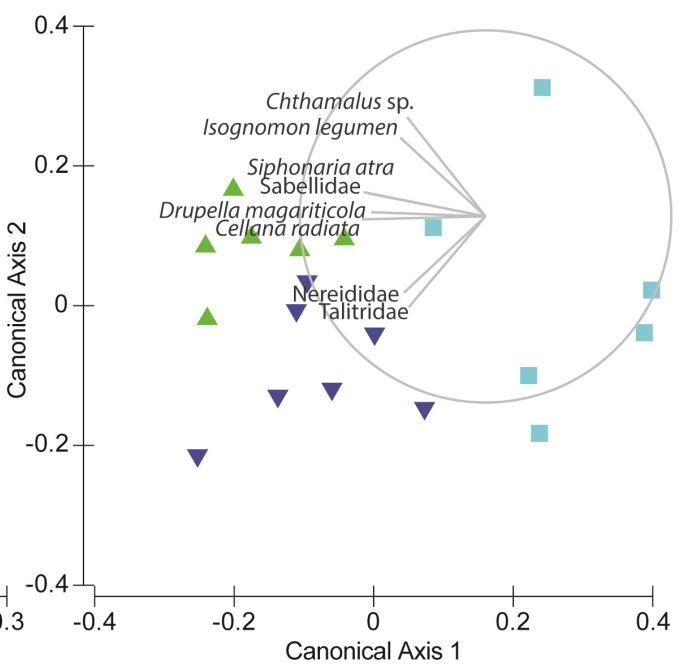

FIGURE $\mathbf{5}$ | Canonical analysis of principal coordinates (CAP) ordination for the tile treatments at each site: (A) Pulau Hantu and (B) Kusu Island; the vector overlay is applied to each plot using Pearson correlation $(\rho=0.6)$ to explore species relationships with the canonical axes.

afford organisms greater shelter from wave action and water movement-potentially a critical resource at sites with higher wave energy such as Kusu Island (Loke and Todd, 2016) - and possibly additional protection from predators.

Although discerning specific niche mechanisms was beyond the scope of this study, differential responses to tile treatments observed among intertidal fauna provides some insights into key abiotic differences between our treatments. For instance, S. guamensis, which was primarily found on uncovered tiles at Kusu Island, was 1-2 orders of magnitude more abundant than all other faunal species. This species tends to dominate exposed flat surfaces (Amnuaypon and Wangkulangkul, 2018; Loke pers. obs.), which may explain why uncovered tiles hosted a greater abundance of $S$. guamensis than the covered tiles (Figure 3A); uncovered tiles had a greater amount of "exposed area." S. guamensis was found to contribute significantly to the site differences in community structure but not to differences in species composition among tile types (Figure 4). This suggests S. guamensis has no strong preference for particular topographical features or substrate material compared to the other gastropods we found at Kusu Island. Conversely, Drupella magariticola, Cellana radiata, and S. atra, were found to be driving the community differences between the concrete tiles (both covered and uncovered tiles) and the granite tiles. This could be due to differences in both substrate material and topographic complexity. Covered and uncovered tiles at Kusu Island were distinguished along the second CAP axis by the pod tree oyster, I. legumen, which usually occurred in clumps on the face beneath the cover (Loke pers. obs). At Pulau Hantu, assemblages colonizing the uncovered and granite tiles were not compositionally distinguishable (Figure 5 and Table 2) but it was evident that covered tiles were distinct from the granite tiles. This is mainly due to Cerithium zonatum and B. amygdalumtostum (bearded ark clams); the latter are usually found on the underside of rocks and boulders on natural shores. Thus, our study shows that, even by adding one more habitat niche (thereby increasing "informational complexity," sensu Loke et al., 2015), we can increase the number of species by providing habitat for specialist species with narrower niche breadths.

While our study demonstrates biodiversity increases from water-retaining features irrespective of surface area, these increases are probably not due to microhabitat differences (niche effects) alone. This is evidenced by the strong positive relationship we observed between the total number of individuals $(N)$ and species richness $(S)$, particularly after accounting for S. guamensis. Had the covered tiles captured a greater species richness but not greater individual abundance compared to the uncovered and granite tiles, it would have shown that the improved richness was due solely to the niche effects provided by the covered tile. Instead, the consistent relationship between $S$ and $N$ suggests that in addition to the niche effects, treatment differences might have simply resulted in the capture of more individuals, which in turn increased the likelihood of capturing more species. While this does not take away from the fact that microhabitat properties such as water retention play an important role in improving species richness, it underscores the importance of accounting for the possible influence of $N$ on $S$ when interpreting results from eco-engineering studies.

\section{CONCLUSION}

In conclusion, water-retaining features combined with complex topography can enhance biodiversity on existing seawalls independently of area through mechanisms that act directly via niche effects, and possibly indirectly via total individual abundance. Until we are able to discern the relative importance of these mechanisms, we suggest that they be explored 
empirically (for instance, by examining species-area relationships (SARs) and/or species-individual relationships) as alternative explanations for the results of future eco-engineering studies. Such efforts are needed to ensure that claims regarding the efficacy of eco-engineering solutions to increase species diversity are justified and well supported.

\section{AUTHOR CONTRIBUTIONS}

LL, TB, and PT designed the experiments. LL conducted the experiment and collected the data. LL and $\mathrm{EH}$ analyzed the data. LL wrote the first draft of the manuscript. All authors contributed to the revisions.

\section{REFERENCES}

Amnuaypon, P., and Wangkulangkul, K. (2018). Influence of habitat modification by rock oysters and barnacles on small-scale distribution of the tropical pulmonate limpet Siphonaria guamensis. Zool. Ecol. 28, 1-8. doi: 10.1080/ 21658005.2018.1520023

Anderson, M., Gorley, R. N., and Clarke, R. K. (2008). Permanova+ for Primer: Guide to Software and Statistical Methods. Plymouth: PRIMER-E Ltd.

Anderson, M. J. (2001). A new method for non-parametric multivariate analysis of variance. Austral. Ecol. 26, 32-46.

Arkema, K. K., Scyphers, S. B., and Shepard, C. (2017). "Living shorelines for people and nature," in Living Shorelines: The Science and Management of Nature-Based Coastal Protection, eds D. M. Bilkovic, M. M. Mitchell, M. K. La Peyre, and J. D. Toft (Boca Raton, FL: CRC Press), 11-30. doi: 10.1201/9781315151465-3

Bertness, M. D., Leonard, G. H., Levine, J. M., and Bruno, J. F. (1999). Climatedriven interactions among rocky intertidal organisms caught between a rock and a hot place. Oecologia 120, 446-450. doi: 10.1007/s004420050877

Bishop, M. J., Mayer-Pinto, M., Airoldi, L., Firth, L. B., Morris, R. L., and Loke, L. H. L. (2017). Effects of ocean sprawl on ecological connectivity: impacts and solutions. J. Exp. Mar. Biol. Ecol. 492, 7-30. doi: 10.1016/j.jembe.2017.01.021

Blockley, D. J. (2007). Effect of wharves on intertidal assemblages on seawalls in Sydney Harbour, Australia. Mar. Environ. Res. 63, 409-427. doi: 10.1016/j. marenvres.2006.10.007

Blockley, D. J., and Chapman, M. G. (2006). Recruitment determines differences between assemblages on shaded or unshaded seawalls. Mar. Ecol. Prog. Ser. 327, 27-36. doi: 10.3354/meps327027

Browne, M. A., and Chapman, M. G. (2011). Ecologically informed engineering reduces loss of intertidal biodiversity on artificial shorelines. Environ. Sci. Technol. 45, 8204-8207. doi: 10.1021/es201924b

Browne, M. A., and Chapman, M. G. (2014). Mitigating against the loss of species by adding artificial intertidal pools to existing seawalls. Mar. Ecol. Prog. Ser. 497, 119-129. doi: 10.3354/meps 10596

Bugnot, A. B., Mayer-Pinto, M., Johnston, E. L., Schaefer, N., and Dafforn, K. A. (2018). Learning from nature to enhance Blue engineering of marine infrastructure. Ecol. Eng. 120, 611-621. doi: 10.1016/j.ecoleng.2018.03.012

Bulleri, F., and Chapman, M. G. (2009). The introduction of coastal infrastructure as a driver of change in marine environments. J. Appl. Ecol. 47, 26-35. doi: 10.1111/j.1365-2664.2009.01751.x

Chapman, M. G., and Blockley, D. J. (2009). Engineering novel habitats on urban infrastructure to increase intertidal biodiversity. Oecologia 161, 625-635. doi: 10.1007/s00442-009-1393-y

Chapman, M. G., and Underwood, A. J. (2011). Evaluation of ecological engineering of "armoured" shorelines to improve their value as habitat. J. Exp. Mar. Biol. Ecol. 400, 302-313. doi: 10.1016/j.jembe.2011.02.025

Cheong, S. M., Silliman, B., Wong, P. P., van Wesenbeeck, B., Kim, C. K., and Guannel, G. (2013). Coastal adaptation with ecological engineering. Nat. Clim. Chang. 3, 787-791. doi: 10.1038/nclimate1854

Connell, J. H. (1972). Community interactions on marine rocky intertidal shores. Annu. Rev. Ecol. Syst. 3, 169-192. doi: 10.1146/annurev.es.03.110172.001125

\section{FUNDING}

This research was funded by National Research Foundation, Prime Minister's Office, Singapore under its Marine Science R\&D Programme (MSRDP- P05), and NParks CME grant number: R-154-000-566-490.

\section{ACKNOWLEDGMENTS}

We would like to thank members of the Experimental Marine Ecology Laboratory for their assistance in the field and Sentosa Development Corporation for facilitating access to the field sites. The comments of two reviewers greatly improved this paper.

Dafforn, K. A., Glasby, T. M., and Airoldi, L. (2015). Marine urbanization: an ecological framework for designing multifunctional artificial structures. Front. Ecol. Environ. 13:82-90. doi: 10.1890/140050

Dyson, K., and Yocom, K. (2015). Ecological design for urban waterfronts. Urban Ecosyst. 18, 189-208. doi: 10.1016/j.jenvman.2016.12.039

Evans, A. J., Firth, L. B., Hawkins, S. J., Morris, E. S., Goudge, H., and Moore, P. J. (2016). Drill-cored rock pools: an effective method of ecological enhancement on artificial structures. Mar. Freshwater Res. 67, 123-130. doi: 10.1071/MF14244

Fahrig, L. (2013). Rethinking patch size and isolation effects: the habitat amount hypothesis. J. Biogeogr. 40, 1649-1663. doi: 10.1111/jbi.12130

Firth, L. B., Browne, K. A., Knights, A. M., Hawkins, S. J., and Nash, R. (2016a). Eco-engineered rock pools: a concrete solution to biodiversity loss and urban sprawl in the marine environment. Environ. Res. Lett. 11:094015. doi: 10.1088/ 1748-9326/11/9/094015

Firth, L. B., White, F. J., Schofield, M., Hanley, M. E., Burrows, M. T., Thompson, R. C., et al. (2016b). Facing the future: the importance of substratum features for ecological engineering of artificial habitats in the rocky intertidal. Mar. Freshw. Res. 67, 131-143. doi: 10.1071/MF14163

Firth, L. B., Schofield, M., White, F. J., Skov, M. W., and Hawkins, S. J. (2014). Biodiversity in intertidal rock pools: informing engineering criteria for artificial habitat enhancement in the built environment. Mar. Environ. Res. 102, 122-130. doi: 10.1016/j.marenvres.2014.03.016

Firth, L. B., Thompson, R. C., White, F. J., Schofield, M., Skov, M. W., and Hoggart, S. P. G. (2013). The importance of water-retaining features for biodiversity on artificial intertidal coastal defence structures. Divers. Distrib. 19, 1275-1283. doi: $10.1111 /$ ddi.12079

Fisher, R. A., Corbet, A. S., and Williams, C. B. (1943). The relation between the number of species and the number of individuals in a random sample of an animal population. J. Anim. Ecol. 12, 42-58. doi: 10.2307/1411

Garrity, S. D. (1984). Some adaptations of gastropods to physical stress on a tropical rocky shore. Ecology 65, 559-574. doi: 10.2307/1941418

Goldberg, N. A., and Foster, M. S. (2002). Settlement and post-settlement processes limit the abundance of the geniculate coralline alga Calliarthron on subtidal walls. J. Exp. Mar. Biol. Ecol. 278, 31-45. doi: 10.1016/S0022-0981(02) 00334-9

Hall, A., Herbert, R. H., Britton, J. R., and Hull, S. (2018). Ecological enhancement techniques to improve habitat heterogeneity on coastal defence structures. Estuar. Coast. Shelf Sci. 210, 68-78. doi: 10.1016/j.ecss.2018.05.025

Heery, E. C., Bishop, M. J., Critchley, L. P., Bugnot, A. B., Airoldi, L., MayerPinto, M., et al. (2017). Identifying the consequences of ocean sprawl for sedimentary habitats. J. Exp. Mar. Biol. Ecol. 492, 31-48. doi: 10.1016/j.jembe. 2017.01.020

Heery, E. C., Dafforn, K. A., Smith, J. A., Ushiama, S., and Mayer-Pinto, M. (2018). Not all artificial structures are created equal: pilings linked to greater ecological and environmental change in sediment communities than seawalls. Mar. Environ. Res. 142, 286-294. doi: 10.1016/j.marenvres.2018.08.012

Hinkel, J., Lincke, D., Vafeidis, A. T., Perrette, M., Nicholls, R. J., Tol, R. S. J., et al. (2014). Coastal flood damage and adaptation costs under 21st century sea-level rise. Proc. Natl. Acad. Sci. U.S.A. 111, 3292-3297. doi: 10.1073/pnas.1222469111 
Huggett, J., and Griffiths, C. L. (1986). Some relationships between elevation, physico-chemical variables and biota of intertidal rock pools. Mar. Ecol. Prog. Ser. 29, 189-197. doi: 10.3354/meps029189

Jaafar, Z., Yeo, D. C. J., and Tan, H. H. (2012). Status of estuarine and marine non-indigenous species in Singapore. Raffles Bull. Zool. 25, 79-92.

Lai, S., Loke, L. H. L., Bouma, T. J., and Todd, P. A. (2018). Biodiversity surveys and stable isotope analyses reveal key differences in intertidal assemblages between tropical seawalls and rocky shores. Mar. Ecol. Prog. Ser. 587, 41-53. doi: 10.3354/meps12409

Loke, L. H. L., Bouma, T. J., and Todd, P. A. (2017). The effects of manipulating microhabitat size and variability on tropical seawall biodiversity: field and flume experiments. J. Exp. Mar. Biol. Ecol. 492, 113-120. doi: 10.1016/j.jembe.2017.01. 024

Loke, L. H. L., Heery, E. C., and Todd, P. A. (2019). "Chapter 26 - Shoreline Defenses," in World Seas: An Environmental Evaluation, 2nd Edn. ed. C. Sheppard (Cambridge, MA: Academic Press), 491-504.

Loke, L. H. L., Jachowski, N. R., Bouma, T. J., Ladle, R. J., and Todd, P. A. (2014). Complexity for artificial substrates (CASU): software for creating and visualising habitat complexity. PLoS One 9:e87990. doi: 10.1371/journal.pone. 0087990

Loke, L. H. L., Ladle, R. J., Bouma, T. J., and Todd, P. A. (2015). Creating complex habitats for restoration and reconciliation. Ecol. Eng. 77, 307-313. doi: 10.1016/ j.ecoleng.2015.01.037

Loke, L. H. L., Liao, L. M., Bouma, T. J., and Todd, P. A. (2016). Succession of seawall algal communities on artificial substrates. Raffles Bull. Zool. 32, 1-10.

Loke, L. H. L., and Todd, P. A. (2016). Structural complexity and component type increase intertidal biodiversity independently of area. Ecology 97, 383-393. doi: 10.1890/15-0257.1

Martins, G. M., Thompson, R. C., Neto, A. I., Hawkins, S. J., and Jenkins, S. R. (2010). Enhancing stocks of the exploited limpet Patella candei d'Orbigny via modifications in coastal engineering. Biol. Conserv. 143, 203-211. doi: 10.1016/ j.biocon.2009.10.004

Metaxas, A., and Scheibling, R. E. (1993). Community structure and organization of tidepools. Mar. Ecol. Prog. Ser. 98, 187-198. doi: 10.3354/meps098187

Morris, R. L., Konlechner, T. M., Ghisalberti, M., and Swearer, S. E. (2018). From grey to green: efficacy of eco-engineering solutions for nature-based coastal defence. Glob. Chang. Biol. 24, 1827-1842. doi: 10.1111/gcb.14063

Narayan, S., Beck, M. W., Reguero, B. G., Losada, I. J., van Wesenbeeck, B., Pontee, N., et al. (2016). The effectiveness, costs and coastal protection benefits of natural and nature-based defences. PLoS One 11:e0154735. doi: 10.1371/ journal.pone.0154735

Neumann, B., Vafeidis, A. T., Zimmermann, J., and Nicholls, R. J. (2015). Future coastal population growth and exposure to sea-level rise and coastal flooding-a global assessment. PLoS One 10:e0118571. doi: 10.1371/journal.pone.0118571

Nicholls, R. J. (2011). Planning for the impacts of sea level rise. Oceanography 24, 144-157. doi: 10.5670/oceanog.2011.34

Nicholls, R. J. (2015). "Chapter 9 - Adapting to sea level rise," in Coastal and Marine Hazards, Risks, and Disasters, eds J. F. Shroder, J. T. Ellis, and D. J. Sherman (Boston, MA: Elsevier), 243-270. doi: 10.1016/B978-0-12-396483-0.00009-1

Perkol-Finkel, S., Hadary, T., Rella, A., Shirazi, R., and Sella, I. (2018). Seascape architecture-incorporating ecological considerations in design of coastal and marine infrastructure. Ecol. Eng. 120, 645-654. doi: 10.1016/j.ecoleng.2017.06. 051

Pioch, S., Relini, G., Souche, J. C., Stive, M. J. F., De Monbrison, D., Nassif, S., et al. (2018). Enhancing eco-engineering of coastal infrastructure with ecodesign: moving from mitigation to integration. Ecol. Eng. 120, 574-584. doi: 10.1016/j.ecoleng.2018.05.034

R Development Core Team (2016). R: A Language and Environment for Statistical Computing. Vienna: R Foundation for Statistical Computing.

Seabra, R., Wethey, D. S., Santos, A. M., and Lima, F. P. (2011). Side matters: microhabitat influence on intertidal heat stress over a large geographical scale. J. Exp. Mar. Biol. Ecol. 400, 200-208. doi: 10.1016/j.jembe.2011.02.010

Strain, E. M., Olabarria, C., Mayer-Pinto, M., Cumbo, V., Morris, R. L., Bugnot, A. B., et al. (2018). Eco-engineering urban infrastructure for marine and coastal biodiversity: which interventions have the greatest ecological benefit? J. Appl. Ecol. 55, 426-441. doi: 10.1111/1365-2664.12961

Tan, W. T., Loke, L. H. L., Yeo, D. C. J., Tan, S. K., and Todd, P. A. (2018). Do Singapore's seawalls host non-native marine molluscs? Aqua. Invasions 13, 365-378. doi: 10.3391/ai.2018.13.3.05

Temmerman, S., Meire, P., Bouma, T. J., Herman, P. M. J., Ysebaert, T., and De Vriend, H. J. (2013). Ecosystem-based coastal defence in the face of global change. Nature 504, 79-83. doi: 10.1038/nature 12859

Underwood, A. J., and Jernakoff, P. (1984). The effects of tidal height, waveexposure, seasonality and rock-pools on grazing and the distribution of intertidal macroalgae in New South Wales. J. Exp. Mar. Biol. Ecol. 75, 71-96. doi: 10.1016/0022-0981(84)90024-8

Waltham, N. J., and Sheaves, M. (2018). Eco-engineering rock pools to a seawall in a tropical estuary: microhabitat features and fine sediment accumulation. Ecol. Eng. 120(A), 1-18. doi: 10.1016/j.ecoleng.2018.05.010

Williams, G. A. (1994). The relationship between shade and molluscan grazing in structuring communities on a moderately-exposed tropical rocky shore. J. Exp. Mar. Biol. Ecol. 178, 79-95. doi: 10.1016/0022-0981(94)90226-7

Zuur, A. F., Ieno, E. N., and Elphick, C. S. (2010). A protocol for data exploration to avoid common statistical problems. Methods Ecol. Evol. 1, 3-14. doi: 10.1111/j. 2041-210X.2009.00001.x

Zuur, A. F., Ieno, E. N., Walker, N. J., Saveliev, A. A., and Smith, G. M. (2009). "Mixed effects modelling for nested data," in Mixed Effects Models and Extensions in Ecology With R, eds A. F. Zuur, E. N. Ieno, N. Walker, A. A. Saveliev, and G. M. Smith (New York, NY: Springer), 101-142. doi: 10.1007/ 978-0-387-87458-6_5

Conflict of Interest Statement: The authors declare that the research was conducted in the absence of any commercial or financial relationships that could be construed as a potential conflict of interest.

Copyright (c) 2019 Loke, Heery, Lai, Bouma and Todd. This is an open-access article distributed under the terms of the Creative Commons Attribution License (CC BY). The use, distribution or reproduction in other forums is permitted, provided the original author(s) and the copyright owner(s) are credited and that the original publication in this journal is cited, in accordance with accepted academic practice. No use, distribution or reproduction is permitted which does not comply with these terms. 